\title{
Transformations and Modern Technology
}

\author{
Jaap Tinbergen \\ Sterrewacht Leiden, Kapteyn Sterrenwacht Roden, Netherlands
}

\begin{abstract}
Transformations are a central issue in making a global network do more than simple monitoring of low-amplitude variability. I explore the approach of observing at a much narrower instrumental bandwidth than is required for the scientific problem; such an approach would have the following advantages:

- transformations can be handled in standard fashion at the instrumental level; at the scientific level, they can be avoided entirely,

- users have almost complete freedom in specifying the shape of the scientific passbands, hence comparison of observational data with stellar atmosphere models can be maximally effective,

- standard star observations can be used repeatedly, for programmes running concurrently in different scientific photometric systems,

- an observer can use existing standard-star observations to create his own specially-tailored photometric system from scratch,

- all-sky homogeneity of the instrumental system can be tested against space photometry such as that provided by HIPPARCOS; this will benefit other scientific systems synthesized from the same instrumental system.

Key components in the hardware will be array detectors with low readout noise and a calibration lamp system designed specifically for this application. A data base provides the link between the observations (in the instrumental system) and the results (with scientific passbands defined by the end user).
\end{abstract}

\section{Introduction}

Young (1992) is the latest author to show how tricky transformations can be, even between photometric systems of roughly the same bandpass. This is a matter we shall have to face in depth when operating global photometric networks. Experience shows that in the course of years passbands always change a little. Equally, nominally identical photometric systems at different sites always differ from each other in at least minor ways. Extensive standard star observations are the only insurance against having to conclude after a number of seasons that the system changed while we were not looking and that, unfortunately, we are not quite sure how it changed. If, with considerable effort, we construct a global photometrictelescope network, should we not spend some thought and engineering effort in 
deciding whether modern technology can help us avoid such pitfalls in future? In this paper I examine what I believe to be key issues in one approach, that of using array detectors to observe at very much narrower bandwidth than the scientific problem really requires, which will allow us to adjust the scientific passbands as we think fit after we have completed our observations. This is in fact spectrophotometry, integrating or convolving to whatever resolution is optimum for the scientific problem in hand; spectroscopists are extending their craft in this direction, but they tend not to emphasize the 'photo' part as much as we should like (e.g. they 'rebin' when convenient, which amounts to a transformation from one photometric system to another).

Observing at narrower bandwidths is not going to solve all our problems. In particular, we should not expect to do better than the best 'monolithic-filter' photometry in absolute flux calibration. However, there would seem to be excellent prospects of limiting transformations to the standard, instrumental, stage of the proceedings (e.g. correcting for equipment drift or for known Doppler shifts). There are bound to be other examples of what can be improved and what cannot. The all-important question is: how much more science can we do by making use of what new technology offers? The answer will depend on how well we can control the transformations from instantaneous to standard instrumental system.

I shall assume that funds and a modest engineering laboratory will be available to a global network consortium (cf. VLBI); to me, there would be no point at all in organising expensive networks of 'cheap and nasty' instruments.

\section{System outline}

In this global-network discussion, I consider a photometer operating on a single star (probably sky simultaneously), but making use of modern technology (see my review of New Techniques):

- Fast telescope of 1-metre class, run by computer programme. Observing schedule determined by 'committee', but up to $50 \%$ of the observing time reserved for standards and calibration lamp exposures, to ensure good quality on behalf of all users.

- Spectrophotometer using a nominally 10-arcsec input diaphragm, a multipassband filter for passband definition, prism spectrometer for physical separation, an array detector to observe $\mathrm{N}$ channels simultaneously ( $\mathrm{N}$ of order 1500), the light of each wavelength channel being spread over $M$ detector pixels (M of order 100; possibly achieved by input scrambler).

- Calibration input simulating the telescope beam, but coming from a local lamp laboratory via a fiber link. The instruments in this lamp laboratory will be crucial to the quality of the spectrophotometry. 
- Archival data base containing, in the instrumental system:

- raw data of programme stars, standards, calibrations, auxiliary quantities,

- data reduced for known and understood instrumental effects, and transformed to an above-atmosphere standard instrumental system.

- Distant scientific user of the data base, feeding back to the 'committee' above.

\section{Transformations}

At the scientific level, there will be no transformations, as passband characteristics will be determined by suitably weighted combinations of the instrumental-system data and can be kept constant. However, the problems noted by Young (1992) will return to plague us at the instrumental level; as Young shows, they do not disappear as we narrow the bands. But at least they can now be tackled by a specialist in a standard and well-documented way, for the benefit of all users (cf synthesis radio-telescopes); this is one potential gain of great significance.

If we have (as we must) a fully-sampled spectrum, we possess all the information needed to correct that same spectrum for out-of-band and high-order-moment contributions. To use the information, we need relative flux calibration of some source or other at all wavelengths within the response of the system. As contributions arise from increasingly distant wavelengths, calibration becomes more uncertain, so the design of the photometer must include sufficiently high blocking for far-out wavelengths. Closer to the central wavelength of a channel, however, the calibration is more certain. This will make things easier compared to doing the transformations in the much wider scientific passbands, in much the same way as the transformation to outside-atmosphere is more reliable for narrower bands. This is another potential gain, of even greater significance (or so I maintain).

Finally, at the instrumental level, extinction treatment will benefit enormously from simultaneous measurements in narrow bands.

\section{Limiting magnitude}

Objections commonly levelled against observing at much narrower bandwidths than required are $\mathrm{A}$ ) that it will take for ever and $\mathrm{B}$ ) that limiting magnitude will suffer badly. These are assumptions, which must be proved and may be disproved.

If we reduce bandwidth by a factor $n$, it will take $n$ times as long to collect the photons, there will be $\mathrm{n}$ bands to observe and photon-limited observations would indeed take very long with a single detector. By observing all bands simultaneously with an array detector, we remove one factor of $n$. Many observations are not photon-limited at all, so that the problem is much less severe than it might seem; it 
should be assessed in detail and set against the advantages of the present approach, such as multiple use of standard star observations.

Deterioration of limiting magnitude also appears to be an unconscious fear rather than solid fact. For accurate point-source spectrophotometry, limiting magnitude (given sufficient photons) is determined by the signal from darkest sky through a focal-plane diaphragm of at least 10 arcsec, or about 17th magnitude; fainter objects rapidly get swamped by photon noise in the sky signal. Let us estimate what combination of telescope aperture, integration time and spectral bandwidth will yield the sufficiency of photons we have just assumed. For a 1-metre telescope, 17th magnitude corresponds to about 1 incident photon per Angstromsecond. If we are to record such a signal without degrading it appreciably by detector readout noise, we should collect of order 100 incident photons per detector pixel (a state-of-the-art CCD has readout noise of about 3 electrons and quantum efficiency of about $50 \%$ ). Assuming that the light in one passband is spread over some 100 pixels for photometric stability, we should integrate for 10000 Angstromseconds. Taking 1000 seconds (cosmic-ray upper limit) for limiting magnitude, we see that minimum bandwidth (FWHM) is of order 10 Angstrom.

This is a very interesting result. Most scientific passbands are of order 200 Angstrom or wider. Since a wavelength range about twice the FWHM contributes to a band and fully sampling the spectrum means spacing the elementary passbands at intervals of half the FWHM, of order 80 instrumental passbands will contribute to a single scientific passband. This is excellent for freedom in shaping the scientific passbands, as in suppressing a conspicuous but irrelevant spectral feature (digital equivalent of a 'notch' filter) or in narrow-band photometry such as H-beta.

Integrating for 1000 secusu at limiting magnitude may sound excessive. But how often does one observe limiting magnitude? Certainly only around New Moon, and certainly NOT for standards and lamp exposures. I do not see this as a serious problem; the technique of 'charge binning' on the CCD chip could probably eliminate the problem altogether while preserving the photometric stability (with a 30 -arcsec diaphragm or 1-electron readout noise, the problem also ceases to exist).

\section{Calibrations}

A large fraction of the engineering effort may have to go into devising suitable calibration systems for our new-fangled photometer. We need two basic kinds of routine calibrations for stability of the system:

- wavelength stability: are the instrumental passbands where we think they are?

- 'gain' (flux sensitivity) of the individual instrumental channels relative to each other (and, less urgently, the time variations).

A suitable selection of standard stars may have to be the final reference for gain; 
between observations of stars on the basic standards network, lamps will have to provide interpolation. It is worth emphasizing that the lamp signal need not stay constant, as long as it changes only in a known way (monitoring at the photometer input by filtered photodiodes?); there is a need for some creative engineering.

The approach to routine wavelength calibration which I should like to see thought out in detail would use a solid Fabry-Perot etalon as calibration component. As in the case of the birefringent filter, only one or two parameters determine its properties; the effect of change in those parameters will be visible in many instrumental channels and the pattern of change will to some degree be specific for each parameter. In such an overdetermined system one can expect substantial gain in correcting for wavelength drifts. The approach is similar to the use of pointing models to improve large telescopes and similar gains may be expected here from creative use of computers. Such a calibration situation could comprise:

- lamp spectrum: 2 parameters (blue/yellow, yellow/red)

- birefringent filter: 1 parameter (phase shift by the basic crystal slice)

- solid F-P: 2 parameters (finesse, spacing)

Our hypothetical system would thus have 5 parameters and regular observations in hundreds of channels, so would be extremely over-determined. In the light of experience we would probably wish to add a few parameters to represent slow changes in CCD spectral sensitivity or flexure of the instrument. The key to good results is a sensible form for the parametrization of the system.

The aim of calibration is to relate the actual instantaneous instrument to a virtual instrument with constant parameters; ideally, the global network will have just one (virtual) reference instrument to which all actual instruments relate. For global network application, portability of the calibration system would be a great advantage. It seems possible to design the lamp and its monitoring diodes, and the F-P, in cabin-luggage format. The other components mostly belong to the photometer and should be left in place. A portable standard CCD system does not seem beyond the realm of possibilities and would allow separate calibration of the spectrophotometer optics and the detector at each site. 'Portable' calibration, if successful, would reduce the network to a single instrument without making use of standard star observations; these would then be available for independent consistency checks.

To determine now and again the exact passband shapes and blocking factors for all the channels, a high-purity monochromator will have to be part of the site installation. We measure simultaneously the relative response of all channels to one wavelength at a time, later we use the response of one channel at a time to all the wavelengths. Since we must use some flux-calibrated source to transform the measured stellar signal at one wavelength into a stray contribution at a channel nominally recording some quite different wavelength, the stray contributions from more distant channels are increasingly uncertain and should therefore be blocked 
sufficiently well for this increased uncertainty not to matter.

It is essential that the lamp beam looks exactly like a beam from the telescope. Spectroscopists know this and designs for suitable optical systems are available.

\section{Operations}

As I see it, a scientific user of the system would lobby for his observing programme to be first accepted and then scheduled optimally. When the semi-automatic system has obtained the data, the user will inspect the (instrumental-system) data corrected (i.e. 'transformed') for extinction and for equipment changes detected by the calibrations. He will then gather the corrected standard star observations of 2 or 3 seasons and set up his private photometric system $a b$ initio by defining the passbands and determining (above-atmosphere) standard-star differences in magnitude and colours, then looking for closure errors and other systematic effects. Having decided what further (partly ad-hoc) corrections to apply, the user will obtain highly accurate results in his private photometric system.

Of course, there will be quicker routes to less-than-optimal results. The distinction will be in how we use the archival data and we do not throw away all possibilities of 'the best' results whenever we decide to optimise for something else rather than accuracy. If only we can agree on what the passbands of the standard systems 'really' are, widespread use of private photometric systems for specialised purposes will not stop us using the same data again for standard systems. We can eat our cake and still have it; paraphrasing one of the other speakers: while peasants guzzle coarse bread, Marie Antoinette can nibble at her Gâteau des Rois, both confections created from the same trough of dough. And that would be a Revolution.

\section{Conclusions}

I have suggested that, for point-source spectrophotometry, it may be possible to avoid the problem of 'transformations' at the scientific level, and to control it more effectively at the instrumental level, by implementing an old concept. The essence of the technique is a posteriori passband control, which seems practicable with the newest generation of CCDs. Many engineering details will need to be solved before one can confidently plan a global-network instrument on this basis, but if we don't set our sights on progress why are we here at all? VLBI provides a valid precedent of organising a single but multi-site facility for a variety of users.

\section{References:}

Young, A.T. 1992, Astron. Astrophys. 257, 366-388 


\section{Discussion}

R. Florentin Nielsen: In your calculations of feasibility you use a read-out-noise of 3 electrons. This is not really the state-of-the art today. CCDs with multiple non-destructive read-outs can have a read-out-noise of $<1$ electron ( $F$. Fanesick).

Tinbergen So much the better, if detailed engineering shows this to be practicable, it will reduce the 1000 s maximum integration time to 100 s, short enough to monitor extinction on a nearby star between 100 s partial exposures. The data-handling problem will not improve, however.

E. Budding: I just wanted to make a comment about your expression "cheap and nasty", which may reflect a certain heretical tendency when set against the leading orthodoxy of market-related policy making. Perhaps we should also be thinking about cheap and attractive(?).

Tinbergen: I was referring to a simple classical photometer, which is cheap but generally produces nasty transformations. I am not against simplicity when it helps. 